\title{
Taste responses in patients with Parkinson's disease
}

\author{
H Sienkiewicz-Jarosz, A Scinska, W Kuran, D Ryglewicz, A Rogowski, E Wrobel, A Korkosz, \\ A Kukwa, W Kostowski, P Bienkowski
}

J Neurol Neurosurg Psychiatry 2005;76:40-46. doi: 10.1136/jnnp.2003.033373

See end of article for authors' affiliations

.....................

Correspondence to: Dr P Bienkowski, Department of

Pharmacology, Institute of Psychiatry and Neurology, Sobieskiego 9 St., 02-957 Warsaw, Poland; bienkow@ipin.edu.pl

Received

26 November 2003

In revised form

7 April 2004

Accepted 9 April 2004

\begin{abstract}
Objective: Preclinical studies indicate that dopaminergic transmission in the basal ganglia may be involved in processing of both pleasant and unpleasant stimuli. Given this, the aim of the present study was to assess taste responses to sweet, bitter, sour, and salty substances in patients with Parkinson's disease (PD).

Methods: Rated intensity and pleasantness of filter paper discs soaked in sucrose (10-60\%), quinine $(0.025-0.5 \%)$, citric acid $(0.25-4.0 \%)$, or sodium chloride $(1.25-20 \%)$ solutions was evaluated in 30 patients with PD and in 33 healthy controls. Paper discs soaked in deionised water served as control stimuli. In addition, reactivity to $100 \mathrm{ml}$ samples of chocolate and vanilla milk was assessed in both groups. Taste detection thresholds were assessed by means of electrogustometry. Sociodemographic and neuropsychiatric data, including cigarette smoking, alcohol consumption, tea and coffee drinking, depressive symptoms, and cognitive functioning were collected.

Results: In general, perceived intensity, pleasantness, and identification of the sucrose, quinine, citric acid, or sodium chloride samples did not differ between the PD patients and controls. Intensity ratings of the filter papers soaked in $0.025 \%$ quinine were significantly higher in the PD patients compared with the control group. No inter-group differences were found in taste responses to chocolate and vanilla milk. Electrogustometric thresholds were significantly $(p=0.001)$ more sensitive in the PD patients.

Conclusions: $\mathrm{PD}$ is not associated with any major alterations in responses to pleasant or unpleasant taste stimuli. Patients with PD may present enhanced taste acuity in terms of electrogustometric threshold.
\end{abstract}

A ccording to the dopamine hypothesis of reward, midbrain dopaminergic neurones constitute a critical part of the brain reward system and mediate the rewarding effects of food, water, sex, and drugs of abuse. ${ }^{1-5}$ In its simplest form, the dopamine hypothesis of reward postulates that the rewarding properties of any stimulus are a direct consequence of dopamine release evoked by this stimulus in the striatum. ${ }^{245}$ In addition, the theory states that dopamine deficits may produce anhedonia, a condition defined as a decreased experience of pleasure after presentation of natural or "chemical" rewards. ${ }^{25-7}$

The dopamine hypothesis of reward prompted some researchers to speculate that patients with idiopathic Parkinson's disease (PD) may show symptoms of anhedonia. ${ }^{2}$ It has been found repeatedly that PD leads to progressive atrophy of both nigrostriatal and mesolimbic dopaminergic pathways. ${ }^{9-11}$ However, experimental evidence linking PD with anhedonia comes from a single study on the rewarding properties of a dopaminergic psychostimulant, methylphenidate. The drug tended to produce weaker rewarding effects in PD subjects compared with healthy controls. ${ }^{12}$ Surprisingly little is known about acute reactivity of PD patients to other chemical or natural rewards. Sweet taste and pleasant odours are considered phylogenetically old natural rewards and their hedonic evaluation is regarded as the indicator of the reward system function. ${ }^{13-16}$ However, any study on hedonic responses to olfactory stimuli in PD could be complicated by the fact that PD patients present severe deficits in odour detection, discrimination, and identification. ${ }^{17-21}$ Taste function in PD has not been assessed in detail as yet. Given these factors, we decided to evaluate taste responses (rated intensity, pleasantness, and identification) in PD patients and in age matched controls. Both groups rated the intensity and pleasantness of filter paper discs soaked in sucrose (sweet), quinine (bitter), citric acid (sour), and sodium chloride (salty) solutions. The subjects were also exposed to $100 \mathrm{ml}$ samples of chocolate and vanilla milk. In order to evaluate perithreshold taste sensitivity, detection thresholds were assessed by means of electrogustometry. ${ }^{22}$

\section{METHODS \\ Participants}

Patients with idiopathic PD, stages I-III according to Hoehn and Yahr, ${ }^{24}$ were taken from a local registry established in the Institute of Psychiatry and Neurology for the Warsaw district. A consecutive series of 35 subjects was invited to participate. All subjects were ambulatory and living with their families. In each case, the diagnosis was confirmed by two neurologists from the study team (WK, HS-J). One patient refused to take part in the study, one was excluded because of cognitive impairment, and three were excluded after neurological examination. Thus, a group of 30 patients ( 17 males, 13 postmenopausal females) was finally recruited for the study. Twenty six of those patients were being treated with L-dopa, 11 with selegiline, two with amantadine, and one with biperiden. The four subjects who were not treated with L-dopa received selegiline $(n=2)$, amantadine $(n=1)$, or selegiline and amantadine in combination $(n=1)$. Patients with clinical fluctuations were examined in the "on" state. Baseline characteristics of the PD group are shown in table 1. A control group was recruited through all institutions involved in the study from families of staff members. Thirty three controls (20 males, 13 post-menopausal females) were selected from a group of 34 subjects. One potential participant was excluded after neurological examination.

Abbreviations: ANOVA, analysis of variance; AUDIT, Alcohol Use Disorder Identification Test; BDI, Beck Depression Inventory; L-dopa, L3,4-dihydroxyphenylalanine; MMSE, Mini Mental State Examination; PD, Parkinson's disease; rCBF, regional cerebral blood flow 


\begin{tabular}{|c|c|c|c|}
\hline Parameter & Controls ( $n=33$ ) & Patients $(n=30)$ & $\mathrm{p}$ \\
\hline Age (years) & $64.0(1.3)$ & $64.0(1.5)$ & 0.68 \\
\hline Women (\%) & 42.4 & 43.3 & 0.96 \\
\hline Married (\%) & 63.3 & 80.0 & 0.56 \\
\hline University degree (\%) & 39.4 & 43.3 & 0.84 \\
\hline Weight (kg) & $73.3(3.0)$ & $73.2(2.8)$ & 0.87 \\
\hline Height $(\mathrm{cm})$ & $170.0(1.8)$ & $170.1(1.6)$ & 0.90 \\
\hline No. of medical conditions* & $1.4(0.3$ & $0.4(0.1)$ & 0.004 \\
\hline \multicolumn{4}{|l|}{ No. of drugs taken } \\
\hline excluding APAs & $1.8(0.3)$ & $0.9(0.20)$ & 0.02 \\
\hline including APAs & $1.8(0.3)$ & $2.5(0.20)$ & 0.11 \\
\hline $\begin{array}{l}\text { Duration of Parkinson's disease (years), } \\
\text { mean (SEM) (range) }\end{array}$ & - & $7.3(0.9)(0.5-16)$ & - \\
\hline $\begin{array}{l}\text { Hoehn and Yahr staget, } \\
\text { mean(SEM) (range) }\end{array}$ & - & $2.1(0.20)(1-3)$ & - \\
\hline $\begin{array}{l}\text { Schwab and England rating } \neq \text {, } \\
\text { mean(SEM) (range) }\end{array}$ & - & $85.8(2.0)(60-100)$ & - \\
\hline $\begin{array}{l}\text { Duration of } \mathrm{L} \text {-dopa treatment (years), } \$ \\
\text { mean (SEM) (range) }\end{array}$ & - & $4.9(0.2)(0.02-12)$ & - \\
\hline $\begin{array}{l}\text { L-dopa daily dose }(\mathrm{mg}) \S \text {, } \\
\text { mean (SEM) (range) }\end{array}$ & - & $572(71)(100-1500)$ & - \\
\hline Sialorrhea (\%) & - & 43.3 & - \\
\hline MMSE score & $28.3(0.3)$ & $28.4(0.3)$ & 0.78 \\
\hline BDI score & $12.7(1.4)$ & $13.2(1.4)$ & 0.64 \\
\hline AUDIT score & $2.7(0.4)$ & $2.4(0.4)$ & 0.54 \\
\hline Current smokers (\%) & 27.3 & 6.7 & 0.07 \\
\hline Tea drinking (cups per day) & $3.9(0.3)$ & $3.3(0.3)$ & 0.22 \\
\hline Coffee drinking (cups per day) & $0.6(0.1)$ & $0.7(0.1)$ & 0.59 \\
\hline $\begin{array}{l}\text { Adding sugar to caffeinated } \\
\text { beverages (spoonful per cup) }\end{array}$ & $1.1(0.1)$ & $1.2(0.2)$ & 0.96 \\
\hline Sweet craving on the day of testing & $1.4(0.3)$ & $2.5(0.5)$ & 0.10 \\
\hline Self reported smell impairment (\%) & & $36.77^{\circ}$ & 0.01 \\
\hline Self reported taste impairment (\%) & 6.1 & 13.3 & 0.37 \\
\hline \multicolumn{4}{|c|}{$\begin{array}{l}\text { Values are expressed as means (SEM) unless otherwise indicated. } \\
\text { The Mann-Whitney U test or the } \chi^{2} \text { test was used for inter-group comparisons. } \\
\text { *The sum of chronic medical conditions other than Parkinson's disease was calculated for each subject. } \\
\text { †Stage 1-11 patients, stage 2-7 patients, stage 3-12 patients. } \\
\text { †Schwab and England. } \\
\text { \$Calculated for the } 26 \text { patients treated with the drug. } \\
\text { Findicates significant inter-group differences. } \\
\text { APAs, anti-parkinsonian agents. }\end{array}$} \\
\hline
\end{tabular}

The subjects in both groups were white, aged $44-75$ years, with no prior history of psychiatric disorders except nicotine dependence, and had had no acute medical conditions over the previous 30 days. Only non-demented subjects whose Mini Mental State Examination (MMSE) scores $^{25}$ were $\geqslant 24$ were included to the study.

The study was carried out in accordance with the Declaration of Helsinki, and the study protocol was reviewed and approved by the Ethics Committee on Human Studies (protocol no. IPiN/13/2001). Each participant read and signed an informed consent form after study procedures had been fully explained. The subjects were paid for their participation (100 PLN $=€ 22$ ).

\section{General design}

A single test session was conducted between 10 am and 12.30 $\mathrm{pm}$ in a quiet, well ventilated, and temperature controlled room. The participants were asked to refrain from eating, drinking, and smoking for at least 1 hour prior to the test session.

The subjects were questioned regarding basic sociodemographic variables, chronic medical conditions and drugs taken, drinking alcohol, coffee, and tea, smoking cigarettes, adding sugar to caffeinated beverages, and subjective taste or smell impairment. Craving for sweets on the day of testing was rated on an 11-point numerical scale $(0=$ "not at all", $10=$ "very much"). The Alcohol Use Disorders Identification Test (AUDIT) ${ }^{26}$ was used to assess alcohol consumption, then the 21 item Beck Depression Inventory (BDI) ${ }^{27-29}$ was completed by each participant.

\section{Preparation of taste samples}

Identical discs ( $1.3 \mathrm{~cm}$ in diameter) were cut from filter paper sheets (Filtrak ${ }^{\circledR}$ no. 388, Spezialpapier-Filtrak GmbH, Post Bärenstein, Germany). Twelve solutions, three for each basic tastant, were prepared with sterile deionised water (Polfa, Lublin, Poland) and stored at room temperature. The paper discs were dipped into the sucrose $(10,25,60 \%$, w/v; Sigma, Poznan, Poland), quinine hydrochloride $(0.025,0.1,0.5 \%$; Polfa), citric acid $(0.25,1.0,4.0 \%$; Sigma $)$, or sodium chloride $(1.25,5.0,20.0 \%$; Polfa) solutions until they were completely soaked. Another set of discs were soaked in deionised water only, to serve as control cues. The filter papers were allowed to dry at room temperature, packed in separate airtight envelopes, and stored at $4^{\circ} \mathrm{C}$. The filter paper methodology was used instead of tastant solutions to avoid olfactory stimulation via the so-called retronasal route, ${ }^{18}$ which could be a confounding factor, as PD has been linked to early diminution of olfactory function..$^{19} 19$

A row of the "sweet", "bitter", "sour", "salty", and "water" discs was prepared for each participant 1 hour before the test and stored at room temperature. Each participant received and rated 13 different filter paper discs. The order of sample administration was counterbalanced across the subjects, although the "bitter" papers were always administered at the end. The $100 \mathrm{ml}$ samples of chocolate and vanilla milk were prepared for each subject from commercially available ultra heat treated milk products (Mlekovita, Wysokie Mazowieckie, Poland). The same volume of deionised water (Polfa) was prepared as another control stimulus.

It should be mentioned that validated tests of taste function are not commercially available. The method used 
in the present study has not been formally assessed in terms of its reliability and validity in a white population. The concentrations of the tastants were selected on the basis of our preliminary studies in a group of adult volunteers. The samples were identified by most of the subjects and intensity ratings varied with concentration (Bienkowski et al, unpublished). The same was true for the groups tested in the present study (see below).

\section{Electrogustometry}

The electrogustometer (TR-06; Rion Co., Ltd., Tokyo, Japan) used in the study is a commercially available device for human taste examination. ${ }^{22}{ }^{23}$ The apparatus allows delivery of anodal currents of low intensity (from $-8 \mathrm{~dB}$ to $34 \mathrm{~dB}$, in $2 \mathrm{~dB}$ steps; 4-400 $\mu \mathrm{A}$ ) at known duration. In the present study, the stimulus duration was kept at $0.5 \mathrm{s.}^{22}{ }^{30}$

The electrogustometer was equipped with a stainless steel, flat, circular stimulus rod (5 $\mathrm{mm}$ in diameter) and a larger indifferent electrode (a neck band). During the test, the stimulus probe was placed on the tongue tip, ${ }^{23}$ while the indifferent electrode was attached to the subject's neck. The participant signalled any new taste sensation on the tongue with the aid of a response button connected to a small buzzer.

\section{Test session}

The taste examination started 15 minutes after completion of the BDI. Each participant was familiarised with all procedures and rating scales before the start of the test.

Firstly, a modified version of initially ascending, single staircase detection threshold procedure was used to assess electrogustometric threshold. The subject was asked to signal any new taste sensation on the tongue by pressing the response button. Care was taken to confirm that each PD patient could really control the button. The current intensity was increased if no response occurred within 3 seconds. The current intensity was decreased (reversal) if the subject signalled detection of the stimulus. The reported threshold (in $\mu \mathrm{A}$ ) was an average of the last four of the six reversals.

Five minutes after completion of electrogustometry, delivery of the filter paper discs started. Each paper was applied on the tongue tip with sterilised tweezers. Neither the experimenter nor the participant was aware of the actual content of the filter papers. The participant was asked to saturate the paper with saliva for $10-15 \mathrm{~s}$ and to taste the liberated tastant within the entire oral cavity. The subject rated taste intensity and pleasantness on 11 point numerical scales labelled at the ends (for intensity $0=$ "not at all" to $10=$ "extremely", and for pleasantness $0=$ "extremely unpleasant" to $10=$ "extremely pleasant"). In addition, the subject was asked to describe the taste of the paper using one of five categories: "sweet", "bitter", "sour", "salty", or "none of the above". "Sweet", "bitter", "sour", and "salty" was assumed to be a correct description of the sucrose, quinine, citric acid, and sodium chloride samples, respectively. "None of the above" was considered a correct descriptor of the neutral paper stimulus. The subjects were not required to perform any test related manual tasks as their responses were registered on the response forms by the experimenter. The testing of each paper disc was followed by a 60 second interval during which the subjects rated and identified the tastant, rinsed their mouths with deionised water, and waited for the next sample. The test was performed by the experimenter blinded to the actual content of the paper discs and no feedback was given to the participants as to the correctness of their taste responses.

Five minutes after presentation of the last filter paper, the participant was asked to drink slowly and taste carefully the $100 \mathrm{ml}$ sample of chocolate milk. The same volume of distilled water then served as the neutral stimulus, and was followed by the sample of vanilla milk. Perceived intensity and pleasantness of the samples was rated on the 11 point scales. The subject was also required to identify the taste of the sample as described above. "Sweet" was assumed to be a correct descriptor of the milk samples. "None of the above" was considered a correct descriptor of the water sample. The testing of each sample was followed by a 120 second interval during which the subjects rated and identified the tastant, rinsed their mouths with deionised water, and waited for the next sample.

\section{Statistics}

The Friedman analysis of variance was used to analyse whether rated intensity of a given tastant varied with its concentration. The Mann-Whitney $U$ test or the $\chi^{2}$ procedure (for non-parametric data) was chosen for inter-group comparisons of sociodemographic parameters and taste responses. The Spearman R test was employed to search for correlations between the clinical parameters (table 1) and those taste responses in the Parkinsonian subjects for which significant inter-group differences had been identified. In addition, correlations between responses to sweet tastes and L-dopa dose and treatment duration were calculated for the PD group. The non-parametric tests were chosen after the Kolmogorov-Smirnov test had revealed that most sociodemographic parameters and taste responses were not normally distributed. A probability level $<0.05$ was considered significant. No correction for multiple comparisons was applied. All statistical analyses were performed with the aid of the Statistica software package for Windows (version 5.0; StatSoft, Tulsa, OK, USA).

\section{RESULTS}

Baseline characteristics of the control and PD group are shown in table 1 . The two groups were similar with respect to age, proportion of men and women, marital status, university education, height, weight, coffee or tea drinking, adding sugar to caffeinated beverages, and sweet craving on the day of testing. The BDI, AUDIT, and MMSE scores did not differ between the groups.

The mean number of chronic medical conditions and drugs taken (excluding PD and the antiparkinsonian medications, respectively) was significantly higher in the controls than in the PD group (table 1). No difference between the groups was observed when the antiparkinsonian drugs were included in the analysis. There was a non-significant trend towards a higher percentage of current smokers in the control group $(\mathrm{p}=0.07)$.

More than one third of the patients reported subjective smell impairment (table 1) compared with only two controls reporting that symptom $(\mathrm{p}=0.01)$. Taste problems were indicated by four PD and two control subjects $(p=0.37)$. Notably, only one PD subject reported isolated taste impairment; the other three patients reported both smell and taste diminution.

Table 2 shows electrogustometric thresholds, taste intensity ratings, and proportions of correct taste identifications in both groups. The patients presented significantly lower electrogustometric threshold compared with the controls. The Friedman one way analysis of variance confirmed that intensity ratings varied with concentration for each tastant including the milk samples $(\mathrm{p}<0.01)$. No inter-group differences were found in terms of perceived intensity of the control cues or of the sucrose, citric acid, sodium chloride, or chocolate or vanilla milk samples $(p>0.05)$. The same was true for rated intensity of the paper discs soaked in the two higher quinine concentrations. The PD group rated the papers soaked in $0.025 \%$ quinine as more intense $(p=0.04)$. In general, the subjects correctly identified most of the taste 


\begin{tabular}{|c|c|c|c|c|c|c|}
\hline & \multicolumn{3}{|c|}{ Intensity (0-10) } & \multicolumn{3}{|c|}{ Correct identification (\%) } \\
\hline & Controls & Patients & $p$ & Controls & Patients & $\mathbf{p}$ \\
\hline \multicolumn{7}{|l|}{ Filter paper discs } \\
\hline $\begin{array}{l}\text { Neutral (water- } \\
\text { soaked papers) }\end{array}$ & $0.5(0.2)$ & $0.8(0.2)$ & 0.19 & 91 & 67 & 0.42 \\
\hline \multicolumn{7}{|l|}{ Sucrose } \\
\hline $10.0 \%$ & $3.9(0.4)$ & $4.0(0.4)$ & 0.96 & 85 & 83 & 0.96 \\
\hline $25.0 \%$ & $6.3(0.4)$ & $6.0(0.5)$ & 0.64 & 88 & 97 & 0.79 \\
\hline $60.0 \%$ & $7.1(0.4)$ & $6.1(0.5)$ & 0.11 & 94 & 93 & 0.99 \\
\hline \multicolumn{7}{|l|}{ Quinine } \\
\hline $0.025 \%$ & $1.9(0.4)$ & $3.2(0.5)^{*}$ & 0.04 & 39 & 60 & 0.34 \\
\hline $0.1 \%$ & $6.2(0.5)$ & $6.9(0.5)$ & 0.30 & 94 & 93 & 0.99 \\
\hline $0.5 \%$ & $8.3(0.4)$ & $7.6(0.5)$ & 0.23 & 97 & 100 & 0.93 \\
\hline \multicolumn{7}{|l|}{ Citric acid } \\
\hline $0.25 \%$ & $2.4(0.3)$ & $3.2(0.4)$ & 0.11 & 64 & 73 & 0.72 \\
\hline $1.0 \%$ & $5.6(0.5)$ & $6.2(0.4)$ & 0.45 & 79 & 70 & 0.76 \\
\hline $4.0 \%$ & $8.4(0.3)$ & $8.2(0.3)$ & 0.33 & 91 & 83 & 0.81 \\
\hline \multicolumn{7}{|l|}{ Sodium chloride } \\
\hline $1.25 \%$ & $3.5(0.5)$ & $3.9(0.5)$ & 0.54 & 64 & 67 & 0.91 \\
\hline $5.0 \%$ & $6.1(0.4)$ & $6.1(0.4)$ & 0.93 & 82 & 80 & 0.95 \\
\hline $20.0 \%$ & $8.4(0.3)$ & $8.2(0.3)$ & 0.80 & 91 & 90 & 0.98 \\
\hline \multicolumn{7}{|l|}{ Liquid samples } \\
\hline Chocolate milk & $6.6(0.3)$ & $6.2(0.4)$ & 0.44 & 94 & 97 & 0.94 \\
\hline Water & $0.8(0.2)$ & $2.2(0.4)$ & 0.06 & 85 & 90 & 0.87 \\
\hline Vanilla milk & $6.5(0.4)$ & $6.6(0.4)$ & 0.93 & 100 & 100 & 1.00 \\
\hline \multicolumn{7}{|c|}{$\begin{array}{l}\text { The electrogustometric threshold ( } \mu \mathrm{A} \text { ) was } 95.6(20.0) \text { for controls and } 27.0(6.5) \text { for patients; } p=0.001 \\
\text { (significant difference). } \\
\text { Intensity ratings are expressed as means (SEM). } \\
\text { Correct identification refers to the percentage of subjects who described the control stimuli as "none", the sucrose } \\
\text { samples as "sweet", the quinine samples as "bitter" etc. "Sweet" was assumed to be the correct answer in the case } \\
\text { of the milk samples. } \\
\text { "Significant inter-group differences. }\end{array}$} \\
\hline
\end{tabular}

samples, with no significant differences between the controls and PD patients $(\mathrm{p}>0.3)$. The Spearman $\mathrm{R}$ test revealed that neither electrogustometric thresholds nor intensity ratings of the $0.025 \%$ quinine papers correlated with the clinical parameters specified in table $1(\mathrm{R}<0.3, \mathrm{p}>0.1)$.

Table 3 shows pleasantness ratings of all tastants. The Mann-Whitney $U$ test indicated that pleasantness ratings did not differ between the two groups $(\mathrm{p}>0.1)$. Taste responses to the sucrose and milk samples recorded for the PD patients did not correlate with L-dopa dose and treatment duration $(\mathrm{R}<0.3, \mathrm{p}>0.1)$.

Table 3 Taste pleasantness in the control and PD group

\begin{tabular}{llll}
\hline & Controls & Patients & $\mathbf{p}$ \\
\hline $\begin{array}{l}\text { Filter paper discs } \\
\text { Neutral (water- }\end{array}$ & $4.7(0.2)$ & $4.4(0.3)$ & 0.70 \\
soaked papers) & & & \\
Sucrose & $7.0(0.3)$ & $6.6(0.2)$ & 0.25 \\
$10.0 \%$ & $7.1(0.3)$ & $7.4(0.3)$ & 0.61 \\
$25.0 \%$ & $7.8(0.3)$ & $7.3(0.2)$ & 0.11 \\
$60.0 \%$ & $4.0(0.3)$ & $3.4(0.3)$ & 0.14 \\
Quinine & $2.1(0.3)$ & $1.8(0.3)$ & 0.35 \\
$0.025 \%$ & $1.3(0.2)$ & $1.4(0.3)$ & 0.89 \\
$0.1 \%$ & $4.6(0.2)$ & $4.9(0.3)$ & 0.43 \\
$0.5 \%$ & $4.6(0.3)$ & $4.4(0.4)$ & 0.67 \\
Citric acid & $3.7(0.5)$ & $4.5(0.5)$ & 0.29 \\
$0.25 \%$ & & & \\
$1.0 \%$ & $4.8(0.3)$ & $4.2(0.3)$ & 0.18 \\
$4.0 \%$ & $4.9(0.4)$ & $4.0(0.3)$ & 0.18 \\
Sodium chloride & $3.8(0.5)$ & $3.0(0.5)$ & 0.37 \\
$1.25 \%$ & & & \\
$5.0 \%$ & $8.6(0.2)$ & $8.1(0.3)$ & 0.20 \\
$20.0 \%$ & $5.1(0.3)$ & $5.5(0.3)$ & 0.61 \\
Liquid samples & $8.0(0.2)$ & $7.8(0.3)$ & 0.85 \\
Chocolate milk & Water & & \\
Vanilla milk & &
\end{tabular}

\section{DISCUSSION}

The two groups recruited for the present study did not differ in terms of several important sociodemographic and biological parameters including age, gender ratio, marital status, university education, height, weight, cognitive status, alcohol related problems, and coffee and tea drinking. Given the hypothesis tested in the study, it should be stressed that adding sugar to caffeinated beverages and sweet cravings on the day of testing were also similar in the two groups. As might be expected, smoking tended to be less frequent in the PD group. In this respect, our findings support previous reports on the negative association between smoking rates and PD. ${ }^{31}{ }^{32}$ It was also not surprising that the number of chronic medical conditions other than PD was lower among the patients. It has been reported that PD is associated with equal (for ischaemic stroke, hypertension, and diabetes mellitus) or lower (for myocardial infarction, coronary artery disease, atrial fibrillation, and cancer) cumulative incidence of various physical illnesses compared with the general population..$^{34}$ The difference in the number of medical conditions fits with the lower number of non-parkinsonian medications taken by the PD group. On the other hand, when the antiparkinsonian drugs were included in the analysis, the PD patients tended to consume more drugs than the controls. It should be noted that all these differences may modify the pattern of taste responses.

Notably, the PD patients and controls did not differ in depressive symptoms as measured by the BDI. ${ }^{27}{ }^{28}$ BDI scores in the present study were similar to those reported previously for PD patients with similar clinical characteristics. ${ }^{35}{ }^{36}$ It has repeatedly been shown that $\mathrm{PD}$ subjects present more depressive features than age matched controls; however, estimates of frequency of depression in PD vary widely, from $2.7 \%$ to $70 \% .{ }^{37-39}$ Hence, although we may not exclude the possibility that other questionnaires would have identified inter-group differences in depressive symptoms, it seems 
reasonable to suggest that depression was not a major factor contributing to the present results.

Contrary to the hypothesis formulated in the Introduction, perceived pleasantness of the sweet samples (sucrose, chocolate milk, and vanilla milk) did not differ between the PD and control group. In addition, hedonic ratings of the other samples (bitter, sour, and salty) were also similar in the PD patients and controls. Thus, it seems that dopaminergic dysfunction in the PD subjects ${ }^{2}$ did not lead to any obvious alteration in perceived pleasantness/aversiveness of gustatory stimuli. Animal studies with the taste reactivity paradigm have revealed that neither dopamine receptor antagonists nor dopamine depletion in the striatum altered appetitive taste responses to sucrose solutions. ${ }^{10-42}$ Moreover, dopamine receptor antagonists fail to diminish the rewarding effects of amphetamine or cocaine in humans. ${ }^{43} 44$

Our results are also in accordance with a recent report of Künig et $a l,{ }^{45}$ who used a simple operant task to measure regional cerebral blood flow ( $\mathrm{rCBF}$ ) in response to monetary reward. PD patients and age matched controls presented distinct patterns of $\mathrm{rCBF}$ increases in response to monetary reward, but the subjective value of earnings did not differ between the groups. The PD group earned slightly less money during the study. ${ }^{45}$ In line with the latter finding, Czernecki et $a .^{46}$ have shown that PD patients were impaired on stimulus-reward learning and reversal in an operant procedure in which points and tones served as symbolic rewards. However, the Parkinsonian group presented normal extinction of responding and actually tended to emit more responses when rewarding feedback was withdrawn. In another study, ${ }^{36}$ PD patients and osteoarthritis controls with comparable degrees of disability did not differ in mean hedonic tone scores on the Snaith-Hamilton Pleasure Scale. ${ }^{47}$ In contrast, the PD group had significantly higher levels of apathy, defined as reduced interest in purposeful behaviours, which were positively correlated with executive impairment. A subgroup of the PD patients with higher levels of apathy showed more anhedonia on the Snaith-Hamilton Pleasure Scale. ${ }^{36}$ The inverse relationship between apathy and executive functioning was also demonstrated in a larger sample of Parkinsonian individuals. ${ }^{48}$

More apathy in PD subjects was also found by Isella et al. ${ }^{8}$ In the same study, the Parkinsonian patients presented mild symptoms of physical anhedonia as measured by the Physical Anhedonia Scale, ${ }^{49}$ but they were also more depressed and demented. The PD group had worse performance in tests of executive function, and physical anhedonia tended to correlate with executive impairment. ${ }^{8}$ Thus, the results of the present and previous studies ${ }^{1} 836454650-52$ may indicate that it is learning and/or motivational deficit (apathy) that impairs processing of rewarding cues in PD and that hedonic tone may be largely unaffected in PD subjects. This notion fits well with theories linking striatal dopaminergic transmission to preparatory phase of motivational behaviours and reward associated learning, but not to subjective pleasure and euphoria. ${ }^{1} 34404243$

Contrary to the data discussed above, it has been reported that PD patients present decreased sensitivity to the rewarding effects of oral methylphenidate. ${ }^{12}$ In that study, two pairs of outliers were eliminated from the PD and control group and the final group size was relatively small ( $\mathrm{n}=10$ subjects/ group). The inter-group differences after that adjustment were still modest and not dose dependent. ${ }^{12}$ Moreover, in an earlier experiment, ${ }^{53}$ only PD subjects with major depression reported less euphoria and activation after intravenous methylphenidate administration. Subjective responses to methylphenidate in PD patients without major depression were similar to those observed in healthy controls. Surprisingly, the rewarding effects of methylphenidate in the depressed subjects without PD remained unaffected..$^{53}$ The latter observation corresponds with reports of other researchers indicating that depressed individuals may even show enhanced reactivity to natural and chemical rewards. Hospitalised, depressed patients rated high concentration sucrose solutions as more pleasant than did non-depressed controls. ${ }^{54}$ Recently, strong positive correlations between severity of major depression and the amphetamine rewarding effects have been reported by Tremblay et al. ${ }^{55}$ Given the above, it may be hypothesised that neither atrophy of dopaminergic neurones in $\mathrm{PD}^{2}{ }^{10}$ nor serotonergic/noradrenergic dysfunction in major depression ${ }^{56}$ is sufficient for the development of clinically relevant anhedonia. Only the combined impairment of the three monoaminergic systems, which is probably present in depressed PD indivi-

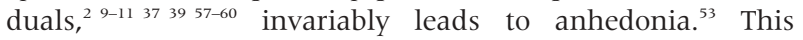
hypothesis needs further validation in multidisciplinary studies.

Notably, the PD group recruited for the present study did not show any major sensory deficit as assessed by intensity ratings and identification of the gustatory samples. Indeed, the PD patients rated the filter papers soaked in the lowest quinine concentration as more intense, compared with the control group. This finding corresponded with lower electrogustometric thresholds observed in the patient group. Neither electrogustometric thresholds nor reactivity to the quinine papers correlated with the basic clinical characteristics of the PD group.

Lower electrogustometric threshold in the PD group does not mean that the patients had enhanced taste sensitivity. It is possible that for some reason threshold taste responses were diminished in the control group; however, our recent results argue against this latter possibility. A mean electrogustometric threshold in a control group (mean age 46.2 years) recruited to another study was similar (102.1 $\mu \mathrm{A}$; Bienkowski et al, unpublished) to that reported in the present study. Lower taste thresholds in the PD group are difficult to reconcile with several reports that olfactory, auditory, visual, and tactile perception may be compromised in PD. ${ }^{17} 192161-67$ In the present study, the percentage of subjects reporting subjective smell impairment was significantly higher in the PD group $(36.7 \%)$ and similar to that reported by other researchers $(28 \%) .^{62}$ In contrast, there was no inter-group difference in the percentage of subjects reporting taste dysfunction. Furthermore, subjective taste impairment in some PD patients could have been secondary to olfactory deficits. ${ }^{68}$ Bearing in mind the functional ${ }^{18}{ }^{69}$ and neuroanatomical ${ }^{15} 18$ interconnections between taste and olfaction, it may be speculated that olfactory deficits in $\mathrm{PD}^{17}{ }^{62}{ }^{64}$ might be compensated by enhanced taste reactivity (like in the present study). However, we did not find any difference in electrogustometric threshold between the PD patients reporting and not reporting olfactory deficits $(\mathrm{p}=0.71)$. Thus, although attractive, the "compensatory" hypothesis needs validation in future studies in which correlations between individual olfactory and taste responses would be calculated.

There is no consensus on oral health status in PD. In one study, PD subjects had significantly more teeth and less caries compared with age matched controls. On the other hand, salivary secretion rate was reduced in a subgroup of patients with more severe parkinsonian symptoms. ${ }^{70}$ More mucositis was also found in the oral cavities of PD patients. ${ }^{71}$ Similarly, a rather inconsistent relationship was observed between PD and dietary habits, although some association with protein rich and carbohydrate rich foods cannot be excluded. ${ }^{72-74}$ The present results may indicate that diminution of taste function is not responsible for altered dietary choices and oral health status in PD. 
Limitations of the present study involve, firstly, some clinical characteristics of the recruited group. All but one PD patients were treated with L-dopa or other dopaminergic therapeutics and all patients were examined in the "on" state. Hence, it could be hypothesised that dopaminergic treatment normalised any pre-existing hedonic deficit in the PD patients. Although we cannot rule out the above hypothesis, it should be borne in mind that L-dopa dose and treatment duration did not predict taste responses to sweet tastes in our patients. Other limitations include the inter-group differences in the number of chronic medical conditions, drugs taken, and cigarette smoking. It should also be borne in mind that the methods used in the study needs further validation and may not reflect all aspects of taste responses in real life conditions. Thus, further research is needed to confirm the present findings.

Concluding, the present results suggest that: (a) PD does not lead to any profound alterations in perceived intensity, pleasantness, or identification of suprathreshold gustatory stimuli, and (b) PD patients may show enhanced taste acuity as assessed by electrogustometry.

\section{ACKNOWLEDGEMENTS}

This work was supported by the State Committee for Scientific Research (KBN grant no. 3P05A 01522 ) and the Institute of Psychiatry and Neurology (grant no. 65/03).

\section{Authors' affiliations \\ H Sienkiewicz-Jarosz, W Kuran, D Ryglewicz, I Department of Neurology, Institute of Psychiatry and Neurology, Warsaw, Poland A Rogowski, E Wrobel, A Korkosz, W Kostowski, P Bienkowski, Department of Pharmacology, Institute of Psychiatry and Neurology, Warsaw, Poland}

A Scinska, A Kukwa, Department of Otolaryngology, Faculty of Dentistry, Warsaw Medical Academy, Warsaw, Poland

W Kostowski, Department of Experimental and Clinical Pharmacology, Faculty of Medicine, Warsaw Medical Academy, Warsaw, Poland

Competing interests: none declared

The first two authors contributed equally to this work.

\section{REFERENCES}

1 Berridge KC, Robinson TE. What is the role of dopamine in reward: hedonic impact, reward learning or incentive salience. Brain Res Rev 1998;28:309-69.

2 Fibiger HC. The neurobiological substrates of depression in Parkinson's disease: a hypothesis. Can J Neurol Sci 1984;11:105-7.

3 Martin-Soelch C, Leenders KL, Chevalley AF, et al. Reward mechanisms in the brain and their role in dependence: evidence from neuropsychological and neuroimaging studies. Brain Res Brain Res Rev 2001;36:139-49.

4 Salamone JD, Correa M. Motivational views of reinforcement: implications for understanding the behavioral functions of nucleus accumbens dopamine. Behav Brain Res 2002;137:3-25.

5 Wise SL, Bozarth MA. A psychomotor stimulant theory of addiction. Psychol Rev 1987;94:469-92.

6 Smith GP. Dopamine and food reward. Prog Psychobiol Physiol Psychol 1995; 16:83-144.

7 Willner P. Animal models of depression: an overview. Pharmacol Ther 1990;45:425-55.

8 Isella V, lurlaro S, Piolti R, et al. Physical anhedonia in Parkinson's disease. J Neurol Neurosurg Psychiatry 2003;74:1308-11.

9 Hornykiewicz $\mathrm{O}$. Neurochemical pathology and the etiology of Parkinson's disease: basic facts and hypothetical possibilities. Mount Sinai J Med 1988;55:11-20.

10 Lang AE, Lozano AM. Parkinson's disease. Part I. NEJM 1998;339:1044-53

11 Uhl GR, Hedreen JC, Price DL. Parkinson's disease: loss of neurons from the ventral tegmental area contralateral to therapeutic surgical lesions. Neurology 1985;35:1215-18.

12 Persico AM, Reich S, Henningfield JE, et al. Parkinsonian patients report blunted subjective effects of methylphenidate. Exp Clin Psychopharmacol 1998;6:54-63.

13 Berridge KC. Measuring hedonic impact in animals and infants: microstructure of affective taste reactivity patterns. Neurosci Biobehav Rev 2000;24: 173-98.

14 Kelley $\mathrm{AE}$, Berridge $\mathrm{KC}$. The neuroscience of natural rewards: relevance to addictive drugs. J Neurosci 2002;22:3306-11.

15 Rolls ET. The orbitofrontal cortex and reward. Cereb Cortex 2000;10:284-94.
16 Scott TR. Taste, feeding and pleasure. Prog Psychobiol Physiol Psychol 1992; 15:231-91.

17 Anasari KA, Johnson A. Olfactory function in patients with Parkinson's disease. J Chronic Dis 1975;28:493-7.

18 Doty RL, ed. Handbook of olfaction and gustation. New York-Basel: Marcel Dekker, Inc, 2003.

19 Hawkes CH, Shepard BC, Daniel SE. Is Parkinson's disease a primary olfactory disorder? Q J Med 1999:92:473-80.

20 Hudry J, Thobois S, Broussolle E, et al. Evidence for deficiencies in perceptual and semantic olfactory processes in Parkinson's disease. Chem Senses 2003;28:537-43

21 Ward CD, Hess WA, Calne BD. Olfactory impairment in Parkinson's disease. Neurology 1983;33:943-6.

22 Kuga $M$, Ikeda $M$, Suzuki K. Gustatory changes associated with the menstrual cycle. Physiol Behav 1999;66:317-22.

23 Miller SL, Mirza N, Doty RL. Electrogustometric thresholds: relationship to anterior tongue locus, area of stimulation, and number of fungiform papillae. Physiol Behav 2002;75:753-7.

24 Hoehn MM, Yahr MD. Parkinsonism: onset, progression and mortality. Neurology 1967;13:427-42.

25 DePaulo JR, Folstein MF. Psychiatric disturbances in neurological patients: detection, recognition, and hospital course. Ann Neurol 1978:4:225-8.

26 Babor TF, Grant M. From clinical research to secondary prevention: international collaboration in the development of the Alcohol Use Disorders Identification Test (AUDIT). Alcohol Health Res World 1989;13:371-4.

27 Beck AT, Ward $\mathrm{CH}$, Mendelson $M$, et al. An inventory for measuring depression. Arch Gen Psychiatry 1961;4:561-71.

28 Beck AT, Steer RA, Garbin MG. Psychometric properties of the Beck Depression Inventory: twenty-five years of evaluation. Clin Psychol Rev 1988;8:77-100.

29 Parnowski T, Jernajczyk W. Beck's Depression Inventory in the rating of mood in normal individuals and in patients with affective disorders. Psychiatria Pol 1977;1 1:417-25.

30 Ajdukovic $\mathrm{D}$. The relationship between electrode area and sensory qualities in electrical human tongue stimulation. Acta Otolaryngol 1984;98:152-7.

31 Fratiglioni L, Wang HX. Smoking and Parkinson's and Alzheimer's disease: review of the epidemiological studies. Behav Brain Res 2000;113:117-20.

32 Jimenez-Jimenez FJ, Mateo D, Gimenez-Roldan S. Premorbid smoking, alcohol consumption, and coffee drinking habits in Parkinson's disease: a case-control study. Mov Disord 1992;7:339-44

33 Levine RL, Jones JC, Bee N. Stroke and Parkinson's disease. Stroke 1992;23:839-42.

34 Vanacore N, Spila-Alegiani S, Raschetti R, et al. Mortality cancer risk in Parkinsonian patients: a population based study. Neurology 1999;52:395-8.

35 Pilo L, Ring H, Quinn N, et al. Depression in multiple system atrophy and in idiopathic Parkinson's disease: a pilot comparative study. Biol Psychiatry 1996;39:803-7.

36 Pluck CG, Brown RG. Apathy in Parkinson's disease. J Neurol Neurosurg Psychiatry 2002;73:636-42.

37 Cummings JL. Depression and Parkinson's disease: a review. Am J Psychiatry 1992;149:443-54.

38 Okun MS, Watts RL. Depression associated with Parkinson's disease: clinical features and treatment. Neurology 2002;58:S63-70.

39 Zesiewicz TA, Hauser RA. Depression in Parkinson's disease. Curr Psychiatry Rep 2002;4:69-73.

40 Berridge KC. Brain substances of liking and wanting. Neurosci Biobehav Rev 1995;20:1-25.

41 Treit D, Berridge KC. A comparison of benzodiazepine, serotonin, and dopamine agents in taste-reactivity paradigm. Pharmacol Biochem Behav 1990:37:451-6.

42 Pecina S, Berridge KC, Parker LA. Pimozide does not shift palatability: separation of anhedonia from sensorimotor suppression by taste reactivity. Pharmacol Biochem Behav 1997;58:801-11.

43 Braver $\mathrm{LH}, \mathrm{De}$ Wit $\mathrm{H}$. High dose pimozide does not block amphetamineinduced euphoria in normal volunteers. Pharmacol Biochem Behav 1997; 56:265-72.

44 Haney M, Ward AS, Foltin RW, et al. Effects of ecopipam, a selective dopamine DI antagonist, on smoked cocaine self-administration by humans. Psychopharmacology 2001;155:330-7.

45 Künig G, Leenders KL, Martin-Sölch C, et al. Reduced reward processing in the brains of Parkinsonian patients. NeuroReport 2000;11:3681-7.

46 Czernecki V, Pillon B, Houeto JL, et al. Motivation, reward and Parkinson's disease: influence of dopatherapy. Neuropsychologia 2002;40:2257-67.

47 Snaith RP, Hamilton M, Morley S, et al. A scale for the assessment of hedonic tone the Snaith-Hamilton Pleasure Scale. Br J Psychiatry 1995;167:99-103.

48 Aarsland D, Larsen JP, Lim NG, et al. Range of neuropsychiatric disturbances in patients with Parkinson's disease. J Neurol Neurosurg Psychiatry 1999;67:492-6.

49 Chapman L, Chapman JP, Raulin ML. Scales for physical and social anhedonia. J Abnormal Psychol 1976;85:374-82.

50 Isella V, Melzi P, Grimaldi M, et al. Clinical, neuropsychological, and morphometric correlates of apathy in Parkinson's disease. Mov Disord 2002;17:366-71.

51 Starkstein SE, Mayberg HS, Preziosi TJ, et al. Reliability, validity and clinical correlates of apathy in Parkinson's disease. J Neuropsychiatry Clin Neurosci 1992:4:134-9.

52 Swainson R, Rogers RD, Sahakian BJ, et al. Probabilistic learning and reversal deficits in patients with Parkinson's disease or frontal or temporal lobe lesions: possible adverse effects of dopaminergic medication. Neuropsychologia 2000;38:569-612. 
53 Cantello R, Aguggia M, Gilli M et al. Major depression in Parkinson's disease and the mood response to intravenous methylphenidate: possible role of the hedonic dopamine synapse. J Neurol Neurosurg Psychiatry 1989;52:724-31.

54 Amsterdam JD, Settle RG, Doty RL, et al. Taste and smell perception in depression. Biol Psychiatry 1987;22:1477-81.

55 Tremblay LK, Naranjo CA, Cardenas L, et al. Probing brain reward system function in major depressive disorder: altered response to dextroamphetamine. Arch Gen Psychiatry 2002;59:409-16.

56 Nelson JC. A review of the efficacy of serotonergic and noradrenergic reuptake inhibitors for treatment of major depression. Biol Psychiatry 1999;46:1301-8.

57 German DC, Manaye KF, White CL, et al. Disease-specific patterns of locus coeruleus cell loss. Ann Neurol 1992;32:667-76.

58 Mayeux R, Stern Y, Cote L, et al. Altered serotonin metabolism in depressed patients with Parkinson's disease. Neurology 1984;34:642-6.

59 Kostic VS, Djuricic BM, Covickovic-Sternic N, et al. Depression and Parkinson's disease: possible role of serotonergic mechanisms. J Neurol 1987;234:94-6.

60 Menza MA, Mark MH. Parkinson's disease and depression: the relationship to disability and personality. Neuropsychiatry Clin Neurosci 1994;6:165-9.

61 Danta G, Hilton RC. Judgment of the visual vertical and horizontal in patients with Parkinsonism. Neurology 1975;25:43-7.

62 Doty RL, Deems D, Stellar S. Olfactory dysfunction in Parkinson's disease: a general deficit unrelated to neurologic signs, disease stage, or disease duration. Neurology 1988;38:1237-44.

63 Gawel MJ, Das P, Vincent S, et al. Visual and auditory evoked potentials in Parkinson's disease. Brain 1978;101:661-71.

64 Quinn NP, Rossor MN, Marsden CD. Olfactory threshold in Parkinson's disease. J'Neurol Neurosurg Psychiatry 1987;50:88-9.
65 Rodnitzky RL. Visual dysfunction in Parkinson's disease. Clin Neurosci 1998;5:102-6.

66 Sakuma K, Nakashima K, Takahashi K. Olfactory evoked potentials in Parkinson's disease, Alzheimer's disease and anosmic patients. Psychiatry Clin Neurosci 1996;50:35-40.

67 Sathian K, Zangaladze A, Green J, et al. Tactile spatial acuity and roughness discrimination: impairments due to aging and Parkinson's disease. Neurology 1997:49: 168-77.

68 Andersson I, Sidenvall B. Case studies of food shopping, cooking and eating habits in older women with Parkinson's disease. J Adv Nurs 2001;35:69-78.

69 Cullen MM, Leopold DA. Disorders of smell and taste. Med Clin N Am 1999;83:57-74.

70 Persson M, Osterberg T, Granerus AK, et al. Influence of Parkinson's disease on oral health. Acta Odontol Scand 1992;50:37-42.

71 Kennedy MA, Rosen S, Paulson GW, et al. Relationship of oral microflora with oral health status in Parkinson's disease. Spec Care Dentist 1994;14:164-8.

72 Abbott RA, Cox M, Markus $H$, et al. Diet, body size and micronutrient status in Parkinson's disease. Eur J Clin Nutr 1992:46:879-84.

73 Chen H, Zhang SM, Hernan MA, et al. Diet and Parkinson's disease: a potential role of dairy products in men. Ann Neurol 2002;52:793-801.

74 Hellenbrand W, Seidler A, Boeing H, et al. Diet and Parkinson's disease. I: A possible role for the past intake of specific foods and food groups. Results from a self-administered food-frequency questionnaire in a case-control study. Neurology 1996;47:636-43

75 Schwab RS, England AC. Projection technique for evaluating surgery in Parkinson's disease. In: Gillingham FJ, Donaldson MC, eds. Third symposium on Parkinson's disease. Edinburgh: Livingstone, 1969. 